\title{
Rigorous Fusion of Gravity Field, Altimetry and Stationary Ocean Models
}

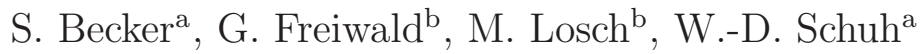 \\ ${ }^{a}$ Institute of Geodesy and Geoinformation, Nussallee 17, 53115 Bonn, Germany \\ ${ }^{b}$ Alfred Wegener Institute for Polar and Marine Research, Postfach 120161, 27515 \\ Bremerhaven, Germany
}

\begin{abstract}
Many characteristics of the ocean circulation are reflected in the mean dynamic topography (MDT). Therefore observing the MDT provides valuable information for evaluating or improving ocean models. Using this information is complicated by the inconsistent representation of MDT in observations and ocean models. This problem is addressed by a consistent treatment of satellite altimetry and geoid height information on an ocean model grid. The altimetric sea surface is expressed as a sum of geoid heights represented by spherical harmonic functions and the mean dynamic topography parameterized by a finite element method. Within this framework the inversion and smoothing processes are avoided that are necessary in step-by-step approaches, such that the normal equations of the MDT can be accumulated in a straightforward way. Conveniently, these normal equations are the appropriate weight matrices for model-data misfits in least-squares ocean model inversions.

Two prototypes of these rigorously combined MDT models, with an associated complete error description including the omission error, are developed for the North Atlantic Ocean and assimilated into a 3D-inverse ocean model.
\end{abstract}


The ocean model solutions provide evidence that satellite observations and oceanographic data are consistent within prior errors.

Keywords: mean dynamic topography, gravity field, altimetry, inverse ocean model, combined model

\section{Introduction}

The ocean's mean dynamic topography (MDT) contains valuable infor3 mation about the ocean circulation (Wunsch and Stammer, 1998). Therefore, 4 estimates of the MDT have the great potential of improving ocean circula5 tion estimates when properly combined with other information, for example, 6 adequate ocean models.

7 In principle, dynamic topography is the difference between the altimet8 ric mean sea surface and geoid height, but calculating this difference is not 9 straightforward because the data types have different representations and spatial resolutions. Gravity field models derived from satellite missions (e.g. GRACE and GOCE) are usually represented by spherical harmonic functions. The downward continuation process from satellite altitude to the Earth's surface leads to an amplification of high frequencies while small scale signals in the smooth field at satellite altitude are hidden in the measurement noise. As a consequence of this unfavorable signal-to-noise ratio, high degree spherical harmonic coefficients in the geoid computation cannot be separated very well. Therefore, the geoid models are typically truncated in a regularization process at a maximum degree $L$ to yield a band-limited representation. The propagated errors of the truncated model, however, only represent the modeled part of the signals (commission error). The part of 
the signal for degrees greater than $L$, that often is omitted, also ought to be taken into account as omission errors to form a consistent model (Losch et al., 2002). In contrast to the geoid information, the altimetric measurements are given as point values or mean values over the footprint of the radar signal along the ground tracks of the satellite. The sample rate along these tracks is very high and the altimetric measurements contain information with high spatial resolution. The sampling is much coarser in the cross track direction, because the ground tracks of the repeating orbit only form a coarse grid.

The two data sets cannot be combined in a straightforward way, because their resolution is different in both space and spectral domain. Special filter processes are introduced (Jekeli, 1981, 1996; Wahr et al., 1998; Swenson and Wahr, 2006; Kusche, 2007) to homogenize all the available information with respect to a least common subspace. Only in this subspace, different phenomena can be compared and hypotheses can be formulated, but the amount of signal lost in such procedures remains unclear. All derived statements are only valid with respect to this subspace, and the geometrical interpretation of the exact content of these filtered quantities is not straightforward. The inherent restriction of altimetry profiles to the ocean with associated boundary issues and the transition from the open ocean to shallow shelves are additional problems that have been identified. For example, Albertella and Rummel (2009) conclude that extending the altimetric data set to the entire globe will inevitably result in a distortion of its spectral content.

To overcome this drawback of filter or smoothing processes a rigorous fusion of the gravity field, altimetric observations, and stationary ocean models is proposed. In this approach, the altimetric sea surface is interpreted as the 
sum of geoid heights represented by spherical harmonic functions and the mean dynamic topography (MDT) parameterized by finite elements. With this combined model the normal equations of the MDT are built directly by a Schur decomposition. Subsequently, these normal equations can be used in stationary ocean models to weight the model data misfit in a least-squares sense. Thus, the inversion of potentially rank deficient covariance matrices and additional smoothing processes that are necessary in step-by-step approaches are avoided.

The paper is organized as follows. In section 2 the interface for the assimilation of the MDT into ocean circulation models is defined and the deterministic and stochastic MDT models are discussed. In section 3 the data sets used in the numerical studies are introduced and first results of the behavior of the estimated MDTs and their accuracy are given. Section 4 shows the results of the integration of the MDT normal equations in the ocean circulation model and discusses the effects of the new data sets on characteristics of the ocean model such as temperature distribution, meridional overturning and heat transports. Conclusions are drawn in section 5 .

\section{Methodology}

In theory, the MDT is the mean sea surface (MSS) referenced to the geoid (N) but a simple combination of MSS and $\mathrm{N}$ data is not straightforward due to the different spatial resolutions and representations of altimetric measurements and a geoid model. Here, the altimetric mean sea surface is interpreted as the sum of geoid height and mean dynamic topography

$$
\operatorname{MSS}(\phi, \lambda)=\mathrm{N}(\phi, \lambda)+\operatorname{MDT}(\phi, \lambda)
$$


where $\phi$, and $\lambda$ are the spherical geocentric coordinates. The geoid is represented as a sum of spherical harmonic functions

$$
\begin{aligned}
& \mathrm{N}(\phi, \lambda)= \\
& \frac{G M}{R \gamma(B)} \sum_{n=0}^{\infty} \sum_{m=0}^{n}\left(\frac{R}{r}\right)^{n+1} \bar{P}_{n m}(\cos \phi)\left(\bar{C}_{n m} \cos (m \lambda)+\bar{S}_{n m} \sin (m \lambda)\right)
\end{aligned}
$$

with the radius vector $r$ depending only on latitude $\phi$, the Earth's radius $R$, the gravitational constant times the Earth's mass $G M$, the geodetic latitude $B$, the normal gravity $\gamma$, the fully normalized Legendre functions $\bar{P}_{n m}(\cos \phi)$ and the Stokes coefficients $\bar{C}_{n m}$ and $\bar{S}_{n m}$.

The mean dynamic topography is represented by a linear combination of finite element basis functions $b_{k}(\phi, \lambda), k \in K$ with a set of indices $K$ that label the basis functions

$$
\operatorname{MDT}(\phi, \lambda)=\sum_{k \in K} a_{k} b_{k}(\phi, \lambda)
$$

In this study we use linear polynomials as basis functions $b_{k}$. The definition of the finite elements, that is, the choice of the basis functions and nodal points, corresponds directly to the ocean circulation model used in section 4 . In this way the mean dynamic topography can be assessed directly on the target grid; the coefficients $a_{k}$ represent the nodal values of the field of interest.

Assembling the spherical harmonic coefficients $\bar{C}_{n m}$ and $\bar{S}_{n m}$ in the vector of unknowns $\boldsymbol{x}_{c s}$ and the coefficients of the linear combination of the finite elements in $\boldsymbol{x}_{F E}$, the observation equations for the altimetric information $\boldsymbol{l}_{\mathrm{MSS}}$ become

$$
\boldsymbol{l}_{\mathrm{MSS}}+\boldsymbol{v}_{\mathrm{MSS}}=\left[\begin{array}{ll}
\boldsymbol{A}_{c s} & \boldsymbol{A}_{F E}
\end{array}\right]\left[\begin{array}{c}
\boldsymbol{x}_{c s} \\
\boldsymbol{x}_{F E}
\end{array}\right]
$$


with the error covariance matrix $\boldsymbol{\Sigma}_{\mathrm{MSS}}$. Then the normal equations for the mean sea surface are constructed as

$$
\left[\begin{array}{ll}
\boldsymbol{A}_{c s}^{T} \boldsymbol{\Sigma}_{\mathrm{MSS}}^{-1} \boldsymbol{A}_{c s} & \boldsymbol{A}_{c s}^{T} \boldsymbol{\Sigma}_{\mathrm{MSS}}^{-1} \boldsymbol{A}_{F E} \\
\boldsymbol{A}_{F E}^{T} \boldsymbol{\Sigma}_{\mathrm{MSS}}^{-1} \boldsymbol{A}_{c s} & \boldsymbol{A}_{F E}^{T} \boldsymbol{\Sigma}_{\mathrm{MSS}}^{-1} \boldsymbol{A}_{F E}
\end{array}\right]\left[\begin{array}{c}
\boldsymbol{x}_{c s} \\
\boldsymbol{x}_{F E}
\end{array}\right]=\left[\begin{array}{l}
\boldsymbol{A}_{c s}^{T} \boldsymbol{\Sigma}_{\mathrm{MSS}}^{-1} \boldsymbol{l}_{\mathrm{MSS}} \\
\boldsymbol{A}_{F E}^{T} \boldsymbol{\Sigma}_{\mathrm{MSS}}^{-1} \boldsymbol{l}_{\mathrm{MSS}}
\end{array}\right],
$$

and in abbreviated form

$$
\left[\begin{array}{cc}
\boldsymbol{N}_{c s}^{\mathrm{MSS}} & \boldsymbol{N}_{c s, F E}^{\mathrm{MSS}} \\
\boldsymbol{N}_{F E, c s}^{\mathrm{MSS}} & \boldsymbol{N}_{F E}^{\mathrm{MSS}}
\end{array}\right]\left[\begin{array}{c}
\boldsymbol{x}_{c s} \\
\boldsymbol{x}_{F E}
\end{array}\right]=\left[\begin{array}{l}
\boldsymbol{n}_{c s}^{\mathrm{MSS}} \\
\boldsymbol{n}_{F E}^{\mathrm{MSS}}
\end{array}\right] .
$$

In this study a static solution of a satellite-derived gravity field model from GRACE or GOCE is used, for which the Stokes coefficients $\bar{C}_{n m}, \bar{S}_{n m}$ and the full variance/covariance matrix $\Sigma_{c s}^{\mathrm{G}}$ are available, and therefore the normal equations

$$
\boldsymbol{N}_{c s}^{\mathrm{G}} \boldsymbol{x}_{c s}=\boldsymbol{n}_{c s}^{\mathrm{G}}
$$

In general, the normal equations of altimetric measurements (6) and the normal equations of the geoid model (7) result from independent observation groups, so that the summation theorem of normal equations can be applied to give

$$
\left[\begin{array}{cc}
\boldsymbol{N}_{c s}^{\mathrm{G}}+\boldsymbol{N}_{c s}^{\mathrm{MSS}} & \boldsymbol{N}_{c s, F E}^{\mathrm{MSS}} \\
\boldsymbol{N}_{F E, c s}^{\mathrm{MSS}} & \boldsymbol{N}_{F E}^{\mathrm{MSS}}
\end{array}\right]\left[\begin{array}{c}
\boldsymbol{x}_{c s} \\
\boldsymbol{x}_{F E}
\end{array}\right]=\left[\begin{array}{c}
\boldsymbol{n}_{c s}^{\mathrm{G}}+\boldsymbol{n}_{c s}^{\mathrm{MSS}} \\
\boldsymbol{n}_{F E}^{\mathrm{MSS}}
\end{array}\right] .
$$

Eliminating the gravity field parameters $\boldsymbol{x}_{c s}$ from these normal equations by using a Schur decomposition (Golub and van Loan, 1983, page 192) provides the normal equations for the mean dynamic topography, in short

$$
\overline{\boldsymbol{N}}_{F E} \boldsymbol{x}_{F E}=\overline{\boldsymbol{n}}_{F E}
$$

These normal equations form a consistent set of information. If all parameters are determined by the observations it is straightforward to solve the 
system and compute the covariance matrix. If only some of the parameters are determined by the observations the system becomes unstable or singular. But this is harmless for the approach in this paper, because an inversion of the normal equation matrix is not required. Instead the normal equations are used directly as weights for the new MDT in the Inverse Finite Element Ocean circulation Model (IFEOM). IFEOM is a stationary inverse model that solves the minimization problem

$$
J=\frac{1}{2} \sum_{i} J_{i} \stackrel{!}{=} \min
$$

that is subject to stationary balances of ocean momentum, energy (potential temperature), salt and mass. The cost function (10) contains contributions from quadratic model-data differences (temperature and salinity from a hydrographic atlas and MDT) weighted by the inverses of their respective error covariances. The contributions $J_{i}$ can also be prior information such as smoothness of the solution. For the relative weighting of the different cost function terms, the hydrographic data is scaled by their annual variance. The resulting weights typically increase with depth where the ocean tends to be quiescent. Towards the open boundary at $4.5^{\circ} \mathrm{N}$, weights are increased in order to constrain the model solution to the first guess in the absence of better information. As the gain of information by the new MDT and its error covariance matrix is to be assessed, all these weights remain unchanged in our experiments to allow for comparison. Details of IFEOM can be found in Sidorenko (2004) and Richter (2010).

In general, the error correlations of the observations are unknown a priori so that most covariances reduce to diagonal matrices. Here IFEOM is 
extended by taking into account the full MDT error covariances

$$
\begin{aligned}
J_{M D T} & =\left(\boldsymbol{x}_{F E}^{\text {data }}-\boldsymbol{x}_{F E}^{\text {model }}\right)^{T} \boldsymbol{\Sigma}_{\boldsymbol{x}_{F E}}^{-1}\left(\boldsymbol{x}_{F E}^{\text {data }}-\boldsymbol{x}_{F E}^{\text {model }}\right) \\
& =\left(\boldsymbol{x}_{F E}^{\text {data }}-\boldsymbol{x}_{F E}^{\text {model }}\right)^{T} \overline{\boldsymbol{N}}_{F E}\left(\boldsymbol{x}_{F E}^{\text {data }}-\boldsymbol{x}_{F E}^{\text {model }}\right)
\end{aligned}
$$

with $\boldsymbol{x}_{F E}^{\text {data }}$ being the "observed" data derived from gravimetry and altimetry and $\boldsymbol{x}_{F E}^{\text {model }}$ being their modeled counterparts. The estimation procedure requires the inverse of the variance/covariance matrix $\Sigma_{\boldsymbol{x}_{F E}}$ for weighting the model-data misfit. This inverse is exactly equal to the normal equation matrix $\overline{\mathbf{N}}_{F E}$, so that the normal equations are directly used within IFEOM.

Unfortunately, the data sets in question are not homogeneous: The altimetric mean sea surface has a spatial resolution that is much higher than that of the geoid model; the spatial resolution of the geoid is homogeneous over the globe but the altimetic measurments are only available on the tracks over the ocean. Therefore the frequency spectrum is split into different domains by the individual observations. This separation is described in the following section and special attention is paid to the infinite-dimensional parameter space of the Stokes coefficients.

\subsection{Observation equations}

In this study the static solution of the latest University-of-Bonn GRACEonly gravity field model ITG-Grace2010s (Mayer-Gürr et al., 2010) is used. It is available up to degree and order 180, corresponding to a half-wavelength of $111 \mathrm{~km}$, with the full variance/covariance information $\Sigma_{\mathrm{ITG}}$. Geoid heights are as accurate as $1 \mathrm{~cm}$ at degree and order 150. Consequently, $L=150$ is chosen to divide the vector of unknowns $\boldsymbol{x}_{c s}^{\mathrm{GRACE}}$ into $\boldsymbol{x}_{c s_{1}}$ respresenting the spherical harmonics up to $L=150$ and the remaining less accurate 
parameters $\boldsymbol{x}_{c s_{2}}$ for degrees 151 to 180 so that

$$
\left[\begin{array}{l}
\boldsymbol{x}_{1}^{\mathrm{ITG}} \\
\boldsymbol{x}_{2}^{\mathrm{ITG}}
\end{array}\right]+\left[\begin{array}{l}
\boldsymbol{v}_{1}^{\mathrm{GRACE}} \\
\boldsymbol{v}_{2}^{\mathrm{GRACE}}
\end{array}\right]=\left[\begin{array}{ll}
\boldsymbol{I} & \mathbf{0} \\
\mathbf{0} & \boldsymbol{I}
\end{array}\right]\left[\begin{array}{l}
\boldsymbol{x}_{c s_{1}} \\
\boldsymbol{x}_{c s_{2}}
\end{array}\right] .
$$

GRACE measurements are assumed not to contribute to the signal beyond degree and order 180 in this study. The mean sea surface is modeled by four groups of spherical harmonics and one for the finite elements. The parameter groups $\boldsymbol{x}_{c s_{1}}, \boldsymbol{x}_{c s_{2}}$ together with the finite elements determine the lower frequencies in analogy to the geoid coefficients. The parameter group $\boldsymbol{x}_{c s_{3}}$ describes a transition domain between the observed and the truncated (omitted) spherical harmonic spectrum. The infinite group $\boldsymbol{x}_{c s_{4}}$ is determined by additional external information (cf. section 2.2). Frequencies $>$ degree 180 are only taken into account in the parameterization of the mean sea surface. In this study the mean sea surface is expanded as a sum of spherical harmonics up to degree and order 240. This choice determines the third group of spherical harmonics $\boldsymbol{x}_{c s_{3}}$. The particular choice of this domain is somewhat arbitrary. Tuning showed that the choice of 240 gave reasonable results and that these results are robust to small variations of this maximum degree. The infinite set of coefficients beyond 240 as a fourth set $\boldsymbol{x}_{c s 4}$ completes the parameter vector. The full representation of MSS is

$$
\boldsymbol{l}_{\mathrm{MSS}}+\boldsymbol{v}_{\mathrm{MSS}}=\left[\begin{array}{lllll}
\boldsymbol{A}_{c s_{1}} & \boldsymbol{A}_{c s_{2}} & \boldsymbol{A}_{c s_{3}} & \boldsymbol{A}_{c s_{4}} & \boldsymbol{A}_{F E}
\end{array}\right]\left[\begin{array}{l}
\boldsymbol{x}_{c s_{1}} \\
\boldsymbol{x}_{c s_{2}} \\
\boldsymbol{x}_{c s_{3}} \\
\boldsymbol{x}_{c s_{4}} \\
\boldsymbol{x}_{F E}
\end{array}\right] .
$$


Gravity field and altimetric observations determine only part of the frequency spectrum, so that additional information based on the smoothness of the potential (Schuh and Becker, 2010) is introduced. Considering the normally distributed random variables for the Stokes coefficients $\mathcal{X}_{c s}^{\text {smooth }} \sim$ $\mathcal{N}\left(\mathbf{0}, \boldsymbol{\Sigma}_{c s}^{\text {smooth }}\right)$ results in the following pseudo-observation equations

$$
\left[\begin{array}{l}
\mathbf{0} \\
\mathbf{0} \\
\mathbf{0}
\end{array}\right]+\left[\begin{array}{l}
\boldsymbol{v}_{1}^{\text {smooth }} \\
\boldsymbol{v}_{2}^{\text {smooth }} \\
\boldsymbol{v}_{3}^{\text {smooth }}
\end{array}\right]=\left[\begin{array}{lll}
\boldsymbol{I} & \mathbf{0} & \mathbf{0} \\
\mathbf{0} & \boldsymbol{I} & \mathbf{0} \\
\mathbf{0} & \mathbf{0} & \boldsymbol{I}
\end{array}\right]\left[\begin{array}{l}
\boldsymbol{x}_{c s_{2}} \\
\boldsymbol{x}_{c s_{3}} \\
\boldsymbol{x}_{c s_{4}}
\end{array}\right] .
$$

Kaula's rule (Kaula, 1966) describes the degree-wise signal content of the gravitational potential coefficients in terms of degree variances

$$
\sigma_{n}^{2}=10^{-10} \frac{2 n+1}{n^{4}} \Rightarrow \sigma_{n m}^{2}=\frac{10^{-10}}{n^{4}}
$$

Thus, the stochastic model results in

$$
\begin{aligned}
\Sigma_{c s}^{\text {smooth }} & =\left[\begin{array}{ccc}
\Sigma_{c s_{2}}^{\text {smooth }} & \mathbf{0} & \mathbf{0} \\
\mathbf{0} & \Sigma_{c s_{3}}^{\text {smooth }} & \mathbf{0} \\
\mathbf{0} & \mathbf{0} & \Sigma_{c s_{4}}^{\text {smooth }}
\end{array}\right] \\
& =\left[\begin{array}{ccc}
\operatorname{diag}\left(\boldsymbol{\sigma}_{n m_{2}}^{2}\right) & \mathbf{0} & \mathbf{0} \\
\mathbf{0} & \operatorname{diag}\left(\boldsymbol{\sigma}_{n m_{3}}^{2}\right) & \mathbf{0} \\
\mathbf{0} & \mathbf{0} & \operatorname{diag}\left(\boldsymbol{\sigma}_{n m_{4}}^{2}\right)
\end{array}\right] .
\end{aligned}
$$

Figure 1 summarizes schematically the frequency domains and the associated parameterizations and accuracies of the individual observation groups. Note, that the domain of the parameter group $\boldsymbol{x}_{c s_{3}}$ is mainly determined by the altimetric observations. This domain is called transfer domain and serves as a buffer between high and low frequency parts of the MSS spectrum. Its 


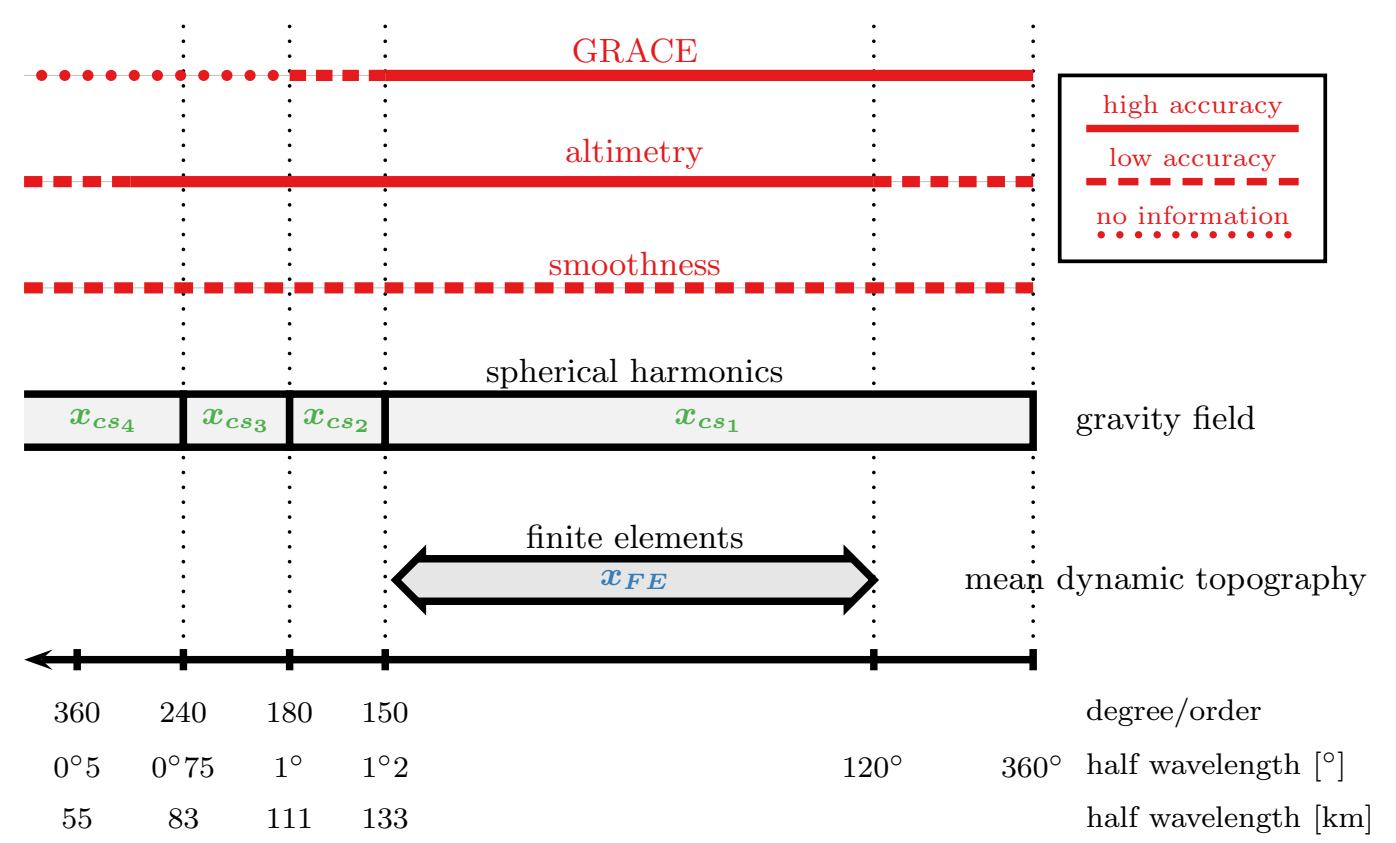

Figure 1: Sketch of frequency domains of different observations their parameterization and accuracy.

purpose is to reduce leakage of the high frequencies of the MSS into the commission domain. The high frequency part of the gravity field is not targeted

\subsection{Parameterization of the infinite-dimensional space}

The altimetric measurements contain frequencies beyond degree and order 240 that ought to be used. The spherical harmonics for this remaining frequency domain up to infinity are collected in the parameter vector $\boldsymbol{x}_{c s_{4}}$ 
and treated separately. Hence, (13) can be recast as

$$
\boldsymbol{l}_{\mathrm{MSS}}+\boldsymbol{v}_{\mathrm{MSS}}=\left[\begin{array}{llll}
\boldsymbol{A}_{c s_{1}} & \boldsymbol{A}_{c s_{2}} & \boldsymbol{A}_{c s_{3}} & \boldsymbol{A}_{F E}
\end{array}\right]\left[\begin{array}{c}
\boldsymbol{x}_{c s_{1}} \\
\boldsymbol{x}_{c s_{2}} \\
\boldsymbol{x}_{c s_{3}} \\
\boldsymbol{x}_{F E}
\end{array}\right]+\boldsymbol{A}_{c s_{4}} \boldsymbol{x}_{c s_{4}}
$$

The extra part of the observation equations $\boldsymbol{A}_{c s_{4}} \boldsymbol{x}_{c s_{4}}$ can now be expressed in terms of random variables

$$
\mathcal{S}=\boldsymbol{A}_{c s_{4}} \mathcal{X}_{c s_{4}} .
$$

The random variable $\mathcal{S}$ is defined by its first two moments, the expectation $\boldsymbol{E}\{\mathcal{S}\}$ and covariances $\boldsymbol{\Sigma}\{\mathcal{S}\}$. In the following, three different choices of the stochastic characteristics of $\mathcal{S}$ are discussed. They respresent two extreme cases, one in which no or only very little prior information is assumed (Rifugio01 and Rifugio02) and one in which the best possible prior information about the omitted signal is used. In the latter case the EGM08 (Pavlis et al., 2008) serves as a place holder or proxy for such information. A realistic assumption is that the omission error probably lies between these extreme cases.

\subsubsection{Approach 1 - Rifugio01}

The first model Rifugio01 assumes no prior information about the signal content of the gravity field beyond degree and order 240 and empirical methods are applied to fill the gap. First, the mean sea surface is deterministically approximated. After subtracting this trend function from the mean sea surface the residual signal is analyzed. This results in an empirical 
auto-covariance function $\operatorname{Cov}_{\mathrm{emp}}\left(\phi, \lambda, \phi^{\prime}, \lambda^{\prime}\right)$ so that the covariance matrix $\Sigma_{S}^{\mathrm{emp}}$ can be assembled. The expectation value of the signal is assumed to be zero due to the reduction by the deterministic model. The stochastic characteristics of $\mathcal{S}$ are

$$
\boldsymbol{E}\{\mathcal{S}\}=0:=\Delta \boldsymbol{l}_{\mathrm{MSS}}, \quad \boldsymbol{\Sigma}\{\mathcal{S}\}=\Sigma_{S}^{\mathrm{emp}}:=\boldsymbol{\Sigma}_{\Delta \mathrm{MSS}}
$$

\subsubsection{Approach 2-Rifugio02}

For the model Rifugio02, smoothness of the gravity field according to Kaula's rule of thumb is introduced as prior information: $\mathcal{X}_{c s_{4}} \sim \mathcal{N}\left(\mathbf{0}, \boldsymbol{\Sigma}_{c s 4}^{\text {smooth }}\right)$. Because the coefficients $\sigma_{n m}^{2}=\frac{1}{2 n+1} \sigma_{n}^{2}$ are not correlated in the model (see section 2.1) the covariance in terms of geoid heights can be written as

$$
\operatorname{Cov}\left(\mathrm{N}(\phi, \lambda), \mathrm{N}\left(\phi^{\prime}, \lambda^{\prime}\right)\right)=
$$

$$
\frac{G^{2} M^{2}}{R^{2} \gamma(B) \gamma\left(B^{\prime}\right)} \sum_{n=241}^{\infty} \frac{R^{2(n+1)}}{\left(r r^{\prime}\right)^{n+1}} \sigma_{n}^{2} P_{n}(\cos \psi)
$$

with the Legendre polynomials $P_{n}(\cos \psi)$ and the spherical distance $\psi$. Denoting the resulting covariance matrix as $\Sigma_{S}^{\text {Kaula }}$ yields for the stochastic characteristics

$$
\boldsymbol{E}\{\mathcal{S}\}=0:=\Delta \boldsymbol{l}_{\mathrm{MSS}}, \quad \boldsymbol{\Sigma}\{\mathcal{S}\}=\Sigma_{S}^{\text {Kaula }}:=\boldsymbol{\Sigma}_{\Delta \mathrm{MSS}}
$$

\subsubsection{Approach 3-Rifugio03}

The gravity field model EGM08 (Pavlis et al., 2008) is available to spherical harmonic degree and order 2160. Here, this information is used to reduce the mean sea surface by the geoid signal of the EGM08 in the range between degree and order 241 and 2160. Beyond 2160 the frequencies are treated in analogy to section 2.2.2. The EGM08 provides error estimates which are used 
to estimate the accuracy of the geoid height information. Based on the error degree variances $\sigma_{n, \mathrm{EGM} 08}^{2}$ the overall covariance information can be written as

$$
\operatorname{Cov}\left(\mathrm{N}(\phi, \lambda), \mathrm{N}\left(\phi^{\prime}, \lambda^{\prime}\right)\right)=
$$

$$
\begin{aligned}
& \frac{G^{2} M^{2}}{R^{2} \gamma(B) \gamma\left(B^{\prime}\right)} \sum_{n=241}^{2160} \frac{R^{2(n+1)}}{\left(r r^{\prime}\right)^{n+1}} \sigma_{n, \mathrm{EGM} 08}^{2} P_{n}(\cos \psi) \\
& \quad+\frac{G^{2} M^{2}}{R^{2} \gamma(B) \gamma\left(B^{\prime}\right)} \sum_{n=2161}^{\infty} \frac{R^{2(n+1)}}{\left(r r^{\prime}\right)^{n+1}} \sigma_{n}^{2} P_{n}(\cos \psi) .
\end{aligned}
$$

Assembling the geoid height information of the EGM08 in the vector $\Delta \boldsymbol{l}_{\text {EGM08 }}$ and the covariances for the respective frequency domains in the matrices $\Sigma_{S 1}^{\mathrm{EGM} 08}$ and $\boldsymbol{\Sigma}_{S 2}^{\text {Kaula }}$ leads to

$$
\begin{aligned}
& \boldsymbol{E}\{\mathcal{S}\}=\Delta \boldsymbol{l}_{\mathrm{EGM} 08}:=\Delta \boldsymbol{l}_{\mathrm{MSS}}, \\
& \boldsymbol{\Sigma}\{\mathcal{S}\}=\Sigma_{S 1}^{\mathrm{EGM} 08}+\Sigma_{S 2}^{\text {Kaula }}:=\Sigma_{\Delta \mathrm{MSS}} .
\end{aligned}
$$

\subsection{The model}

Finally, the complete observation equations for the altimetric measurements are

$$
\overline{\boldsymbol{l}}_{\mathrm{MSS}}+\boldsymbol{v}_{\mathrm{MSS}}=\left[\begin{array}{llll}
\boldsymbol{A}_{c s_{1}} & \boldsymbol{A}_{c s_{2}} & \boldsymbol{A}_{c s_{3}} & \boldsymbol{A}_{F E}
\end{array}\right]\left[\begin{array}{l}
\boldsymbol{x}_{c s_{1}} \\
\boldsymbol{x}_{c s_{2}} \\
\boldsymbol{x}_{c s_{3}} \\
\boldsymbol{x}_{F E}
\end{array}\right] .
$$

Here, $\overline{\boldsymbol{l}}_{\mathrm{MSS}}=\boldsymbol{l}_{\mathrm{MSS}}-\Delta \boldsymbol{l}_{\mathrm{MSS}}$ and $\overline{\boldsymbol{\Sigma}}_{\mathrm{MSS}}=\boldsymbol{\Sigma}_{\mathrm{MSS}}+\boldsymbol{\Sigma}_{\Delta \mathrm{MSS}}$ are different for each of the three previous approaches. The overall gravity field observation 


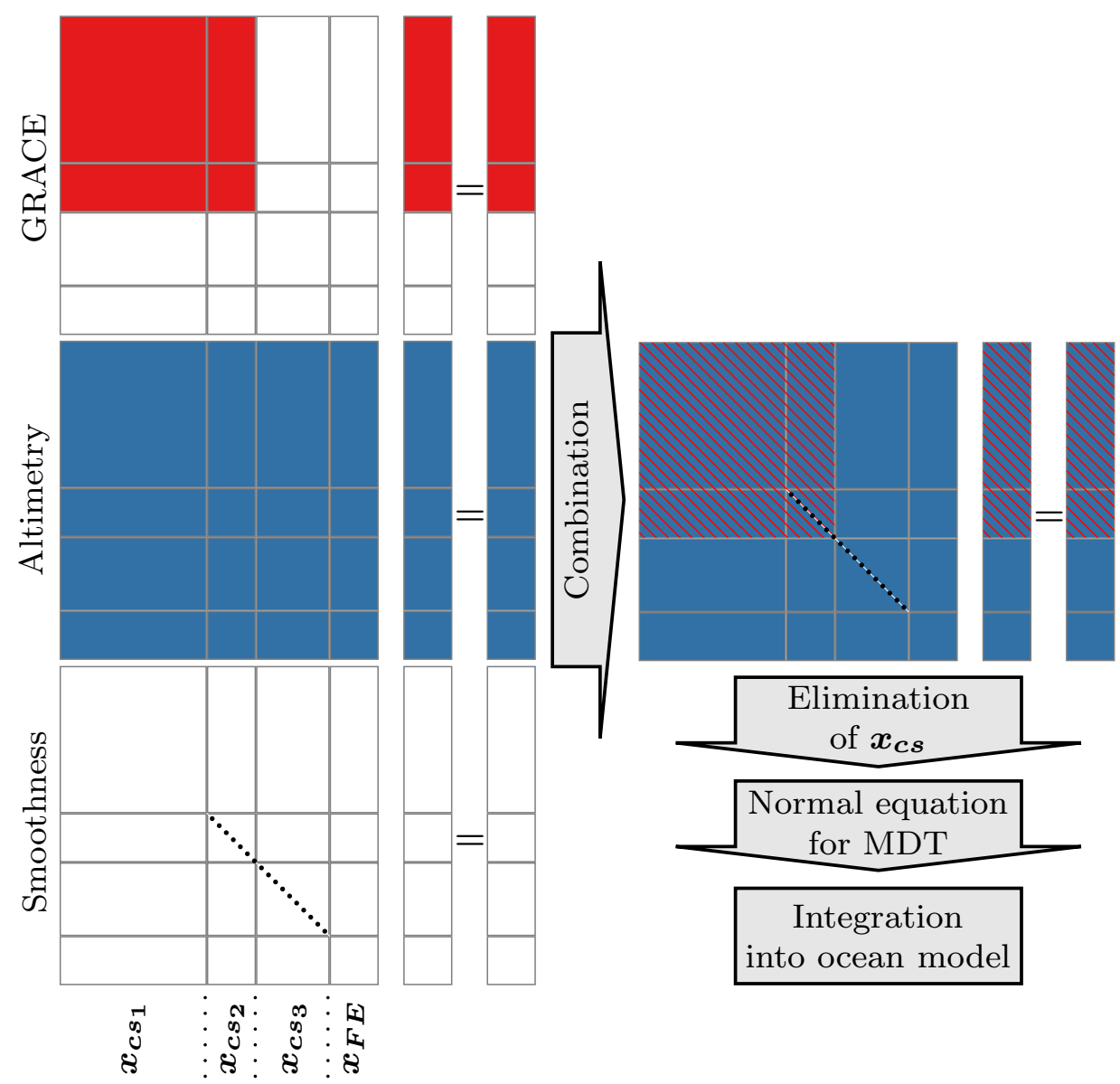

Figure 2: Schematic of the particular normal equations and the rigorous combination model 
equations are

271

$$
\left[\begin{array}{l}
\boldsymbol{x}_{1}^{\mathrm{ITG}} \\
\boldsymbol{x}_{2}^{\mathrm{ITG}}
\end{array}\right]+\left[\begin{array}{l}
\boldsymbol{v}_{1}^{\mathrm{GRACE}} \\
\boldsymbol{v}_{2}^{\mathrm{GRACE}}
\end{array}\right]=\left[\begin{array}{llll}
\boldsymbol{I} & \mathbf{0} & \mathbf{0} & \mathbf{0} \\
\mathbf{0} & \boldsymbol{I} & \mathbf{0} & \mathbf{0}
\end{array}\right]\left[\begin{array}{l}
\boldsymbol{x}_{c s_{1}} \\
\boldsymbol{x}_{c s_{2}} \\
\boldsymbol{x}_{c s_{3}} \\
\boldsymbol{x}_{F E}
\end{array}\right]
$$

with the covariance matrix $\Sigma_{\text {ITG }}$. The pseudo-observation equations for the introduced smoothness conditions are

$$
\left[\begin{array}{l}
\mathbf{0} \\
\mathbf{0}
\end{array}\right]+\left[\begin{array}{l}
\boldsymbol{v}_{1}^{\text {smooth }} \\
\boldsymbol{v}_{2}^{\text {smooth }}
\end{array}\right]=\left[\begin{array}{llll}
\mathbf{0} & \mathbf{1} & \mathbf{0} & \mathbf{0} \\
\mathbf{0} & \mathbf{0} & \mathbf{1} & \mathbf{0}
\end{array}\right]\left[\begin{array}{l}
\boldsymbol{x}_{c s_{1}} \\
\boldsymbol{x}_{c s_{2}} \\
\boldsymbol{x}_{c s_{3}} \\
\boldsymbol{x}_{F E}
\end{array}\right]
$$

with the stochastic information contained in

$$
\Sigma_{c s}^{\text {smooth }}=\left[\begin{array}{cc}
\operatorname{diag}\left(\boldsymbol{\sigma}_{n m_{2}}^{2}\right) & \mathbf{0} \\
\mathbf{0} & \operatorname{diag}\left(\boldsymbol{\sigma}_{n m_{3}}^{2}\right)
\end{array}\right] .
$$

In contrast to (14) which describes the complete observation equations, the parameters $\boldsymbol{x}_{c s_{4}}$ are no longer required here. Because the $\boldsymbol{x}_{c s_{4}}$ are separated from the vector of unknowns, additional smoothness conditions need not be applied in the corresponding frequency domain.

Subsequently the normal equations for the particular groups of observations can be accumulated. Figure 2 shows a schematic diagram of the resulting normal equations. After renaming the coefficients and the right-hand side of equation (8) these are written as

$$
\left[\begin{array}{cc}
\boldsymbol{N}_{c s} & \boldsymbol{N}_{c s, F E} \\
\boldsymbol{N}_{F E, c s} & \boldsymbol{N}_{F E}
\end{array}\right]\left[\begin{array}{c}
\boldsymbol{x}_{c s} \\
\boldsymbol{x}_{F E}
\end{array}\right]=\left[\begin{array}{c}
\boldsymbol{n}_{c s} \\
\boldsymbol{n}_{F E}
\end{array}\right] .
$$


The gravity field parameters $\boldsymbol{x}_{c s}$ can be eliminated by a Schur decomposition from these normal equations to provide the normal equations for the mean dynamic topography

$$
\left(\boldsymbol{N}_{F E}-\boldsymbol{N}_{F E, c s} \boldsymbol{N}_{c s}^{-1} \boldsymbol{N}_{c s, F E}\right) \boldsymbol{x}_{F E}=\left(\boldsymbol{n}_{F E}-\boldsymbol{N}_{F E, c s} \boldsymbol{N}_{c s}^{-1} \boldsymbol{n}_{c s}\right)
$$

$$
\boldsymbol{\Sigma}_{F E}^{-1} \boldsymbol{x}_{F E}=\overline{\boldsymbol{n}}_{F E}
$$

At this point the great advantage and benefit of the rigorous combination model becomes clear. As mentioned above the finite elements are directly applied to the nodal points of the ocean circulation model. Thus the resulting normal equation matrix represents the inverse covariance matrix $\Sigma_{F E}^{-1}$ of the mean dynamic topography required by the ocean circulation model; that is, no additional inversion is required to compute a weight matrix from an error covariance matrix and the MDT can be directly combined with the ocean circulation model.

\section{Numerical results}

The static gravity field solution ITG-Grace2010s (Mayer-Gürr et al., 2010) and the altimetric mean sea surface model MSS_CNES_CLS10 (MSS_CNES_CLS10, 2010) are used in this study. The MSS is given on a regular grid with a resolution of $(1 / 30)^{\circ}$ covering the global oceans between the latitudes $80^{\circ} \mathrm{S}$ and $84^{\circ} N$. MSS_CNES_CLS10 includes an error estimate $\boldsymbol{\Sigma}_{\mathrm{MSS}}$ that mainly reflects the formal errors of the optimal interpolation method used in mapping. Thus, the formal errors may not account for other errors such as radial orbit errors. In this experiment it is assumed that these additional errors are very small compared to the stochastic characteristics of the random field $\mathcal{S}$, 


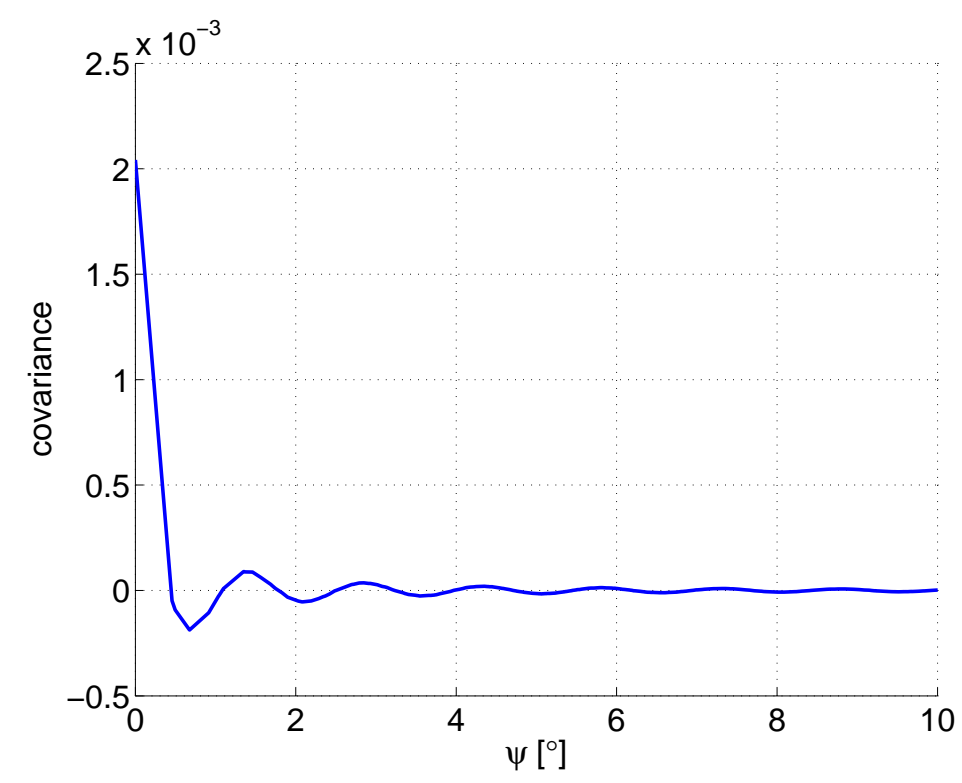

Figure 3: Covariance function for approach Rifugio03.

expressed by $\boldsymbol{\Sigma}_{\Delta \text { MSS }}$ (cf. table 1$)$. The MSS as well as the respective errors for the North Atlantic Ocean are extracted from the original data set. To reduce the computational effort only values on a $0.5^{\circ} \times 0.5^{\circ}$ grid are selected. Figure 3 shows that for the covariance function following approach Rifugio03 (section 2.2.3, equation (22)) point values at a distance of $0.5^{\circ}$ can be considered as nearly uncorrelated because the central maximum falls off very quickly. This observation also holds for the covariance models obtained by approaches Rifugio01 and Rifugio02. Thus, correlations of MSS errors are neglected in this study and a diagonal covariance matrix $\boldsymbol{\Sigma}_{\Delta \mathrm{MSS}}$ is used so that the overall covariance matrix for the MSS results in

$$
\overline{\boldsymbol{\Sigma}}_{\mathrm{MSS}}=\boldsymbol{\Sigma}_{\mathrm{MSS}}+\sigma^{2} \boldsymbol{I} .
$$



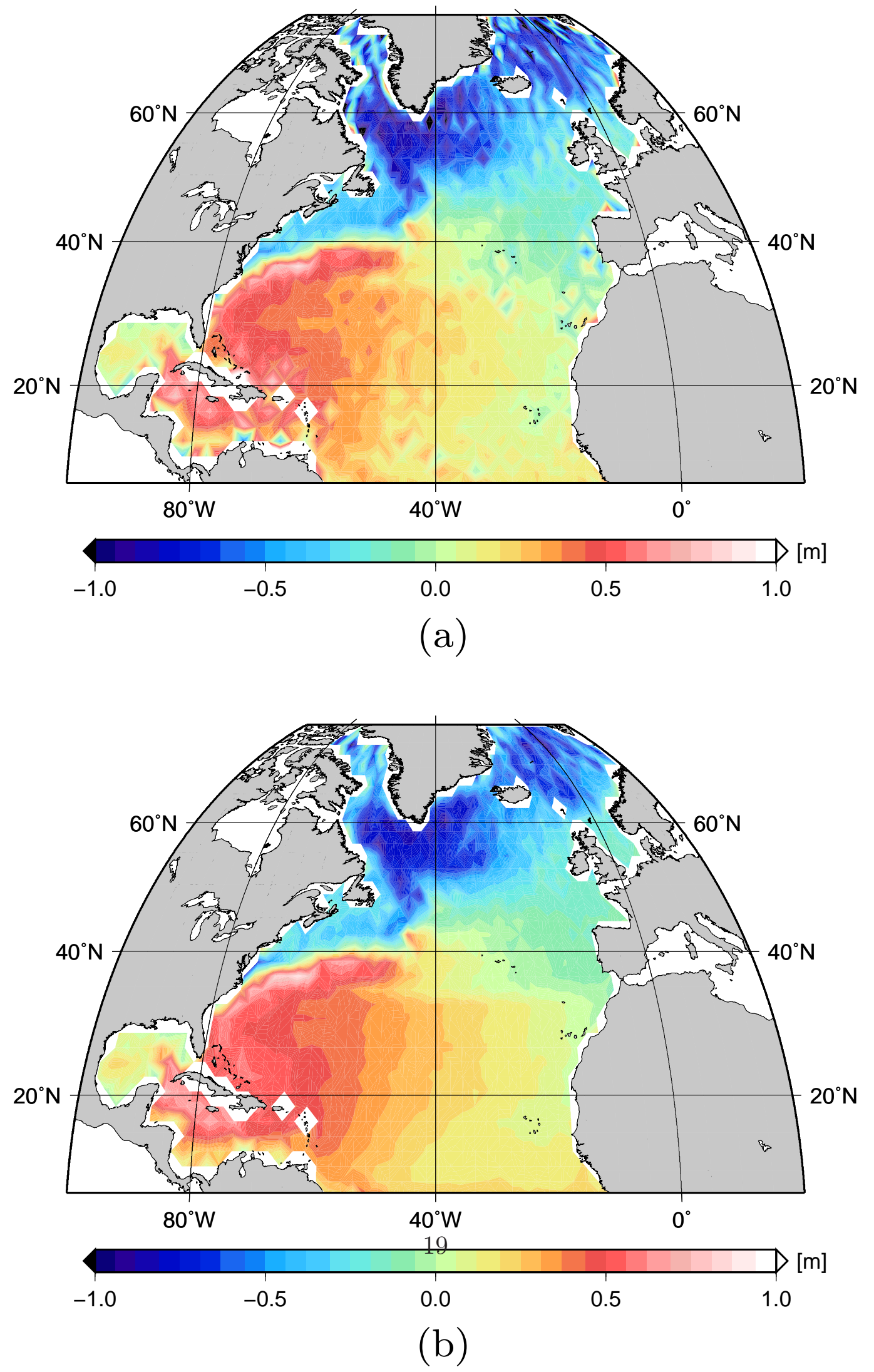

Figure 4: Mean dynamic topographies for approach Rifugio01 (a) and Rifugio03 (b). 


\begin{tabular}{lcc} 
& $\sigma[\mathrm{m}]$ & $\sigma_{\text {total }}[\mathrm{m}]$ \\
\hline \hline Rifugio01 & $\sigma_{\text {emp }}= \pm 0.385 \mathrm{~m}$ & $\pm 0.386 \mathrm{~m}$ \\
\hline Rifugio02 & $\sigma_{\text {Kaula }}= \pm 0.265 \mathrm{~m}$ & $\pm 0.266 \mathrm{~m}$ \\
\hline Rifugio03 $\sqrt{\sigma_{\text {EGM08 }}^{2}+\sigma_{\text {Kaula }}^{2}}=\sqrt{ \pm 0.034^{2} \mathrm{~m}^{2}+ \pm 0.029^{2} \mathrm{~m}^{2}}= \pm 0.045 \mathrm{~m}$ & $\pm 0.048 \mathrm{~m}$
\end{tabular}

Table 1: Standard deviations obtained with different approaches and representative values for the overall standard deviations.

The errors of the MSS_CNES_CLS10 representing the $\boldsymbol{\Sigma}_{\text {MSS }}$ range between $0.3 \mathrm{~cm}$ and $9.69 \mathrm{~cm}$. The resulting standard deviations $\sigma$ obtained by the different approaches as well as a representative value for the overall standard deviation are listed in table 1.

The finite elements are used on a triangulated $2^{\circ} \times 2^{\circ}$ grid with continuous linear polynomials as basis functions. No additional smoothness conditions are applied. The definition of this coarse grid ensures that (29) is solvable and an analysis and a comparison of different mean dynamic topographies is possible in this study. As a prerequisite the spatial resolution of the finite elements has to cover the frequency range for that both the GRACE geoid model and the altimetric mean sea surface provide information with high accuracy (see figure 1). Figure 4 shows the mean dynamic topography for Rifugio01 and Rifugio03. As expected, the Rifugio01 solution is less smooth, probably because the high frequencies in the altimetric measurements leak into the solution. Because of the consistent treatment of signal and omission error, however, the resulting standard deviations also increase with the descreased smoothness of the solution, as shown in figure 5. For a section along longitude 


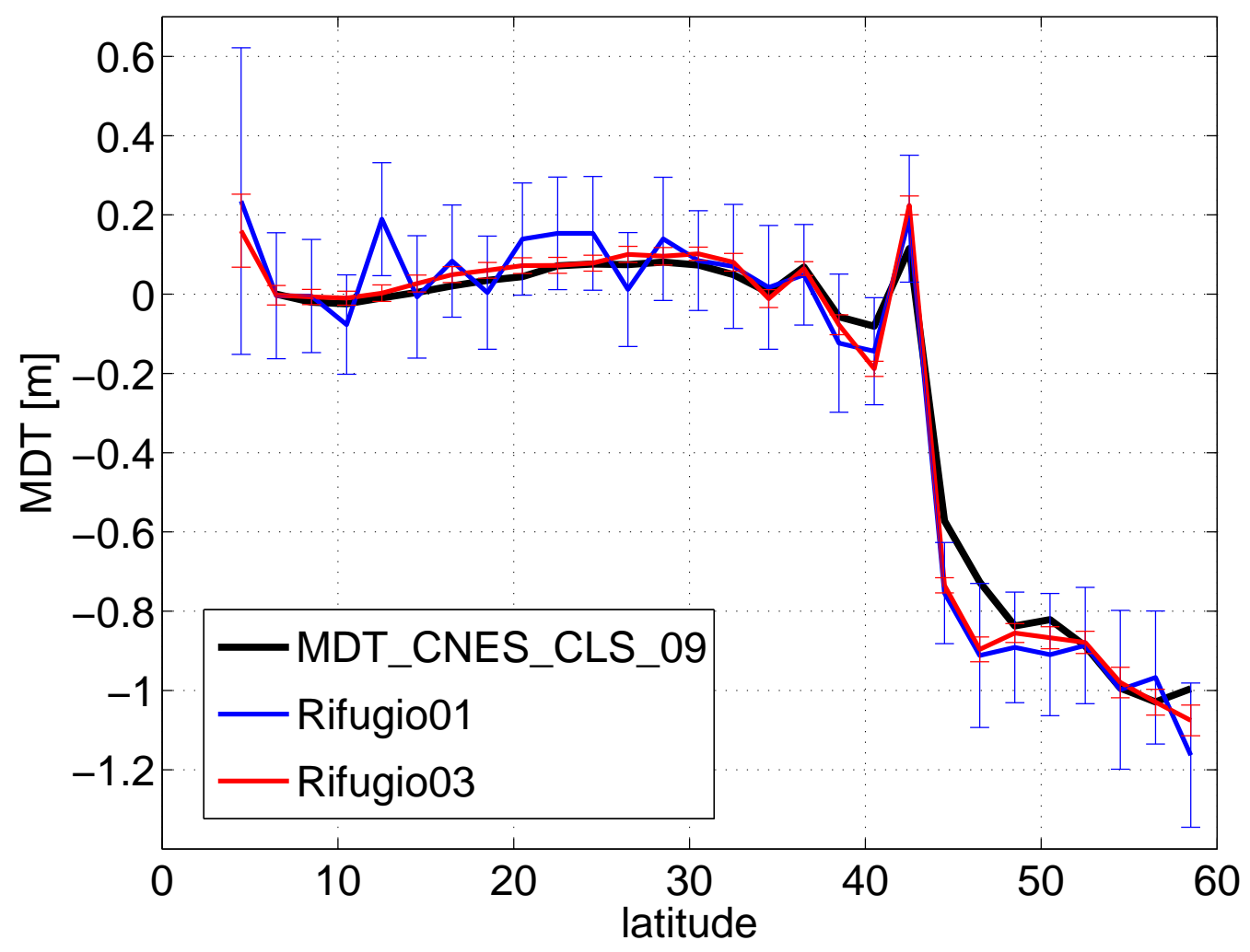

Figure 5: MDT along longitude $-45.5^{\circ}$ with error bars.

$-45.5^{\circ}$, the mean dynamic topography agrees mostly within the corresponding error bars with the mean dynamic topography model MDT_CNES_CLS09 (MDT_CNES_CLS09, 2009).

Figure 6 shows a histogram of differences between the MDT_CNES_CLS09 estimate and Rifugio01 and Rifugio03 (gray bars). For Rifugio03 the distribution has a sharp peak near zero and the root-mean-square (rms) difference is $0.1072 \mathrm{~m}$. For the Rifugio01 solution the distribution of differences is much broader (outliers, that are found mostly near the coast lines, are not shown for clarity) with an rms-difference of $0.2231 \mathrm{~m}$. 

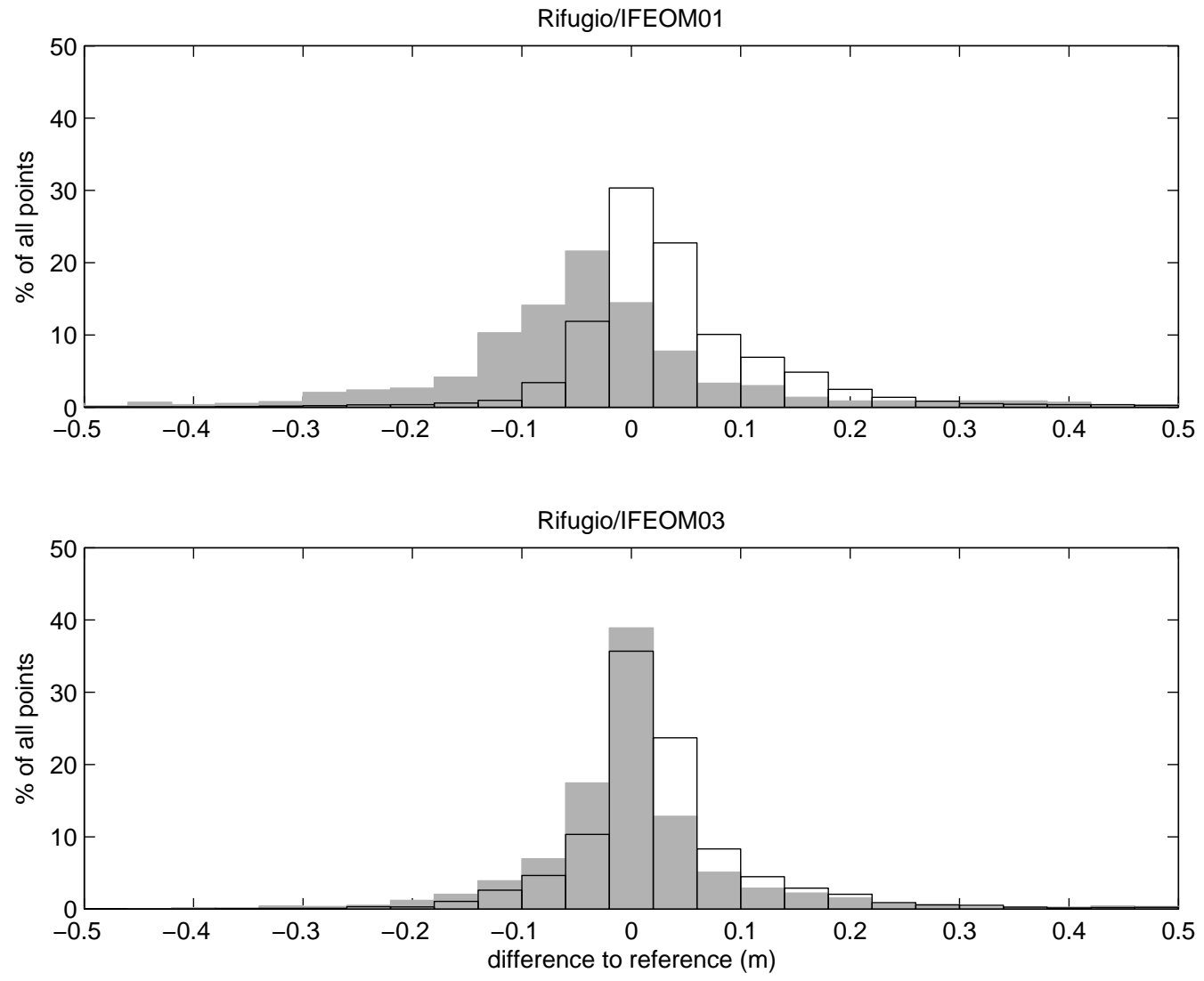

Figure 6: Histogram of difference to MDT estimate by CLS Space Oceanography Division. The gray bars show the difference between of Rifugio01 (top) and Rifugio03 (bottom) solutions to the MDT_CNES_CLS09 estimate (MDT_CNES_CLS09, 2009). The black lines indicate the differences of the corresponding IFEOM solutions after combination with the Rifugio estimates. 
The effects of integrating the different solutions into the Inverse Finite Element Ocean Model (IFEOM) are described in the following section.

\section{Integration into IFEOM}

\subsection{Optimization by IFEOM}

The MDT solutions Rifugio01 and Rifugio03 are combined with the Inverse Finite Element Ocean Model (IFEOM) as described in section 2. The results are labeled IFEOM01 and IFEOM03. The two MDT estimates represent the two extremes: for the estimate Rifugio01 minimal prior information was assumed - the omitted signal has the expectation zero with a large empirical error variance (section 2.2.1); in contrast, the estimate Rifugio03 was obtained by assuming maximal prior information about the omission errorthe omitted signal is assumed to be estimated by EGM08 (section 2.2.3). The resulting estimate is not only smoother for Rifugio03 than for Rifugio01 (as discussed in section 3, figures $4 \mathrm{a}$ and $4 \mathrm{~b}$ ), but also the error estimate is much smaller for Rifugio03 (figure 5) so that the ocean model estimate IFEOM03 is closer to Rifugio03 than IFEOM01 to Rifugio01.

Figure 6 shows that in spite of the large errors (small weights in the cost function) in Rifugio01 the resulting IFEOM estimate (IFEOM01) matches the MDT_CNES_CLS09 estimate better than the Rifugio01 estimate; the corresponding rms-difference is almost as small as that for Rifugio03: $0.1079 \mathrm{~m}$. The small errors (large weights in the cost function) of Rifugio03 make the IFEOM03 solution adjust closely to Rifugio03 so that in the histogram of differences to the MDT_CNES_CLS09 estimate there is only a small change in the bias. The rms-difference is only slightly reduced to $0.0964 \mathrm{~m}$. This 
implies that IFEOM adds information (from other data sources such as hydrography) to the inaccurate estimate Rifugio01 to improve the MDT, while IFEOM can barely change the more accurate estimate Rifugio03.

Figure 7 shows the two MDT estimates IFEOM01 and IFEOM03. The optimization procedure rejects, based on the prior error estimates, the small scale structures still apparent in figures $4 \mathrm{a}$ and $4 \mathrm{~b}$ as unphysical noise, so that both IFEOM estimates are smooth. This is interpreted as a success of the consistent error description of Rifugio01 and Rifugio03.

In the following the solutions IFEOM01 and IFEOM03 are compared to previous solutions by Richter (2010). Richter obtained his solutions by assimilating the Rio05 MDTRio and Hernandez (2004) and sea level anomalies provided by Aviso (Archiving, Validation and Interpretation of Satellites Oceanographic data, www.aviso.oceanobs.com). In the absence of any error estimation for these data, he used an annual variability (variance) in the weighting procedure. This weighting approach is not comparable to the methods described here, but Richter's solutions still provide a well tuned baseline for plausiblity comparisons.

\subsection{Influence of the new data combination on oceanographic features}

The IFEOM03 solution has some remarkable new features that are a consequence of both the new gravity field data and the new combination method. Large differences in temperature compared to a solution of Richter (2010) can be found in the Gulf Stream area. The temperatures at $120 \mathrm{~m}$ depth are higher at the southern flank of the current and lower at the northern side so that the across-stream temperature difference is increased by up to $10^{\circ} \mathrm{C}$ (figure $8 \mathrm{a}$ ). In contrast, the corresponding salinity difference is de- 

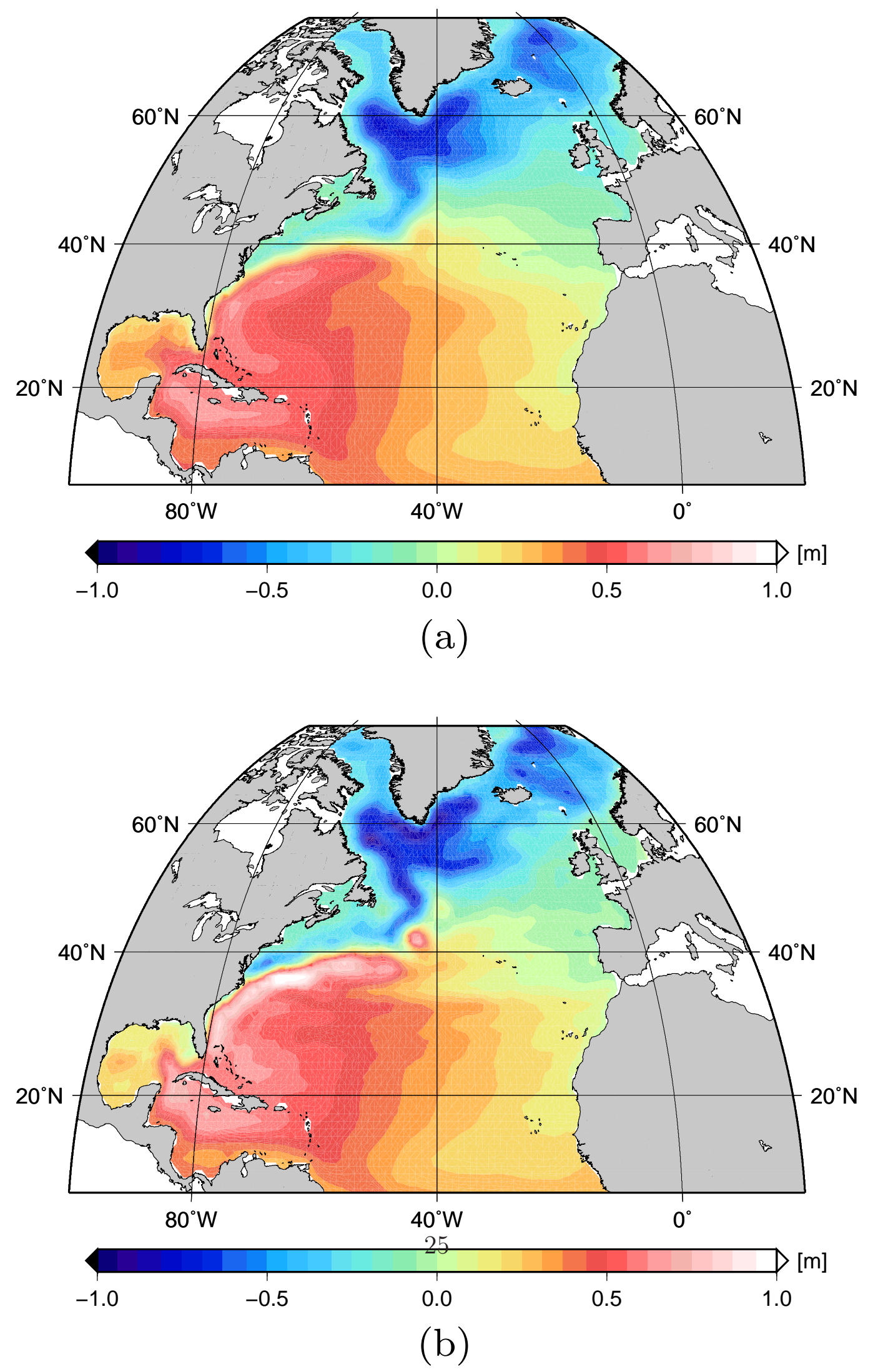

Figure 7: MDT estimates by IFEOM with Rifugio01: IFEOM01 (a) and Rifugio03: IFEOM03 (b). 

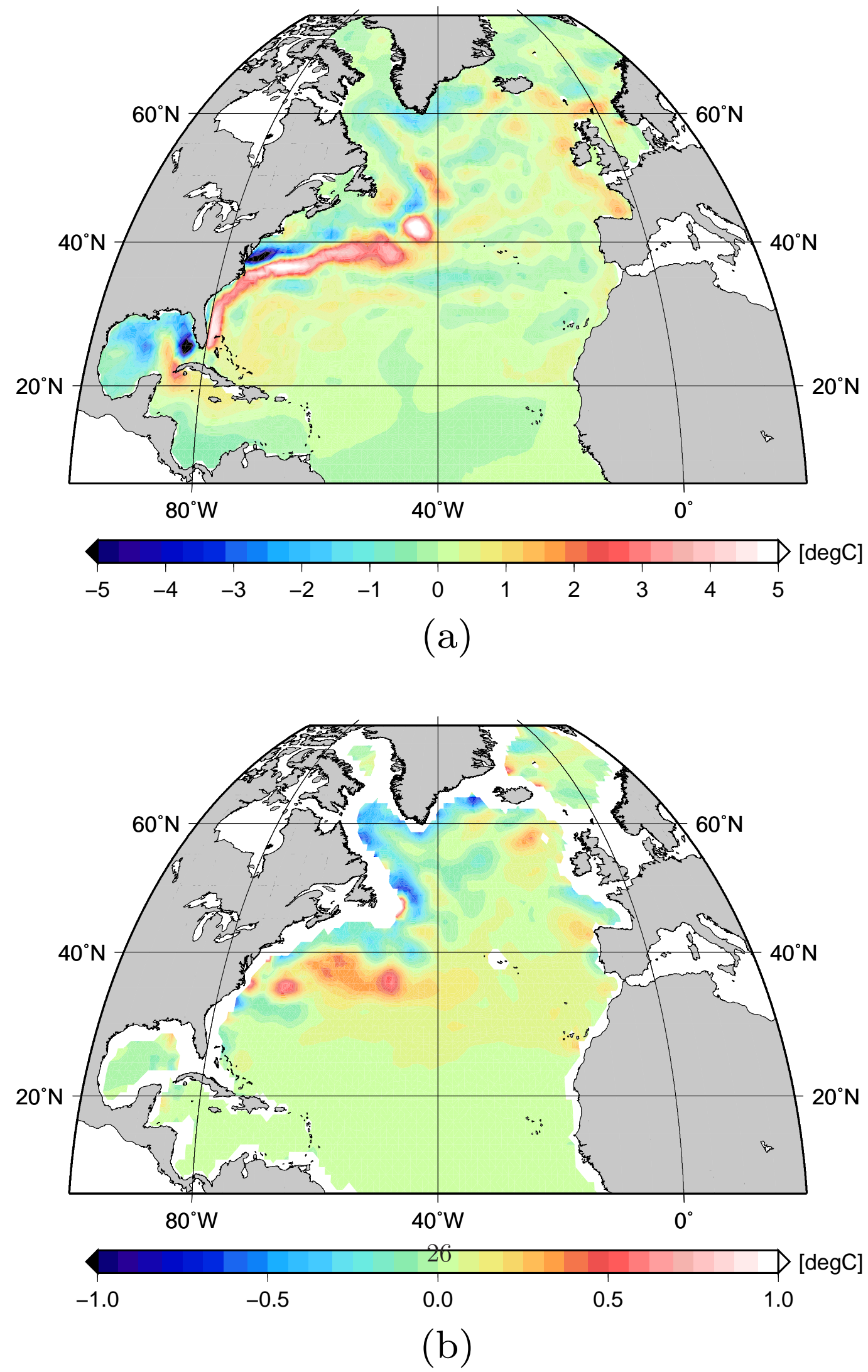

Figure 8: Horizonal maps of temperature difference between IFEOM03 and Richter (2010) solutions at depths $120 \mathrm{~m}$ (a) and at $1000 \mathrm{~m}$ (b). 
creased by about $1^{1}$, so that, compared to the previous solution by Richter, water is less saline at the southern boundary of the Gulf Stream (not shown).

At $1000 \mathrm{~m}$ depth, deep water masses along the coast of Greenland and in the Labrador Sea are approximately $0.5^{\circ} \mathrm{C}$ cooler (figure $8 \mathrm{~b}$ ) and 0.1 more saline (not shown) in the IFEOM03 solution than in the Richter solution. These differences are within the range of the assumed prior errors in this model region of $2.48^{\circ} \mathrm{C}$ and 0.35 , respectively. This finding can be interpreted as an increase in deep water formation rates when more cold and saline surface water sinks to greater depths.

These characteristics are barely visible in the IFEOM01 solution (not shown), because the large errors of Rifugio01 allow only small adjustments to the MDT estimate thereby avoiding deviations from the first guess. The first guess is a long term IFEOM model run on an extended model domain without any satellite altimetry information, but only hydrographic data (Richter, 2010).

The meridional overturning stream function (figure 9) has changed under the influence of the new MDT estimates. Both solutions show a new maximum of over $20 \mathrm{~Sv}\left(1 \mathrm{~Sv}=10^{6} \mathrm{~m}^{3} \mathrm{~s}^{-1}\right)$ near $40-45^{\circ} \mathrm{N}$ that is missing in Richter's solution. The meridional circulation of the IFEOM01 solution is weaker at the Southern boundary compared to Richter's solution. This is also apparent in the integrated meridional heat transports (figure 10). The IFEOM03 solution is affected by the Rifugio03 MDT mostly in the Northern part of the model area.

\footnotetext{
${ }^{1}$ We use the pratical salinity scale (PSS) for values of salinity. Note that in oceanography, salinity is a conductivity ratio and therefore does not have units.
} 

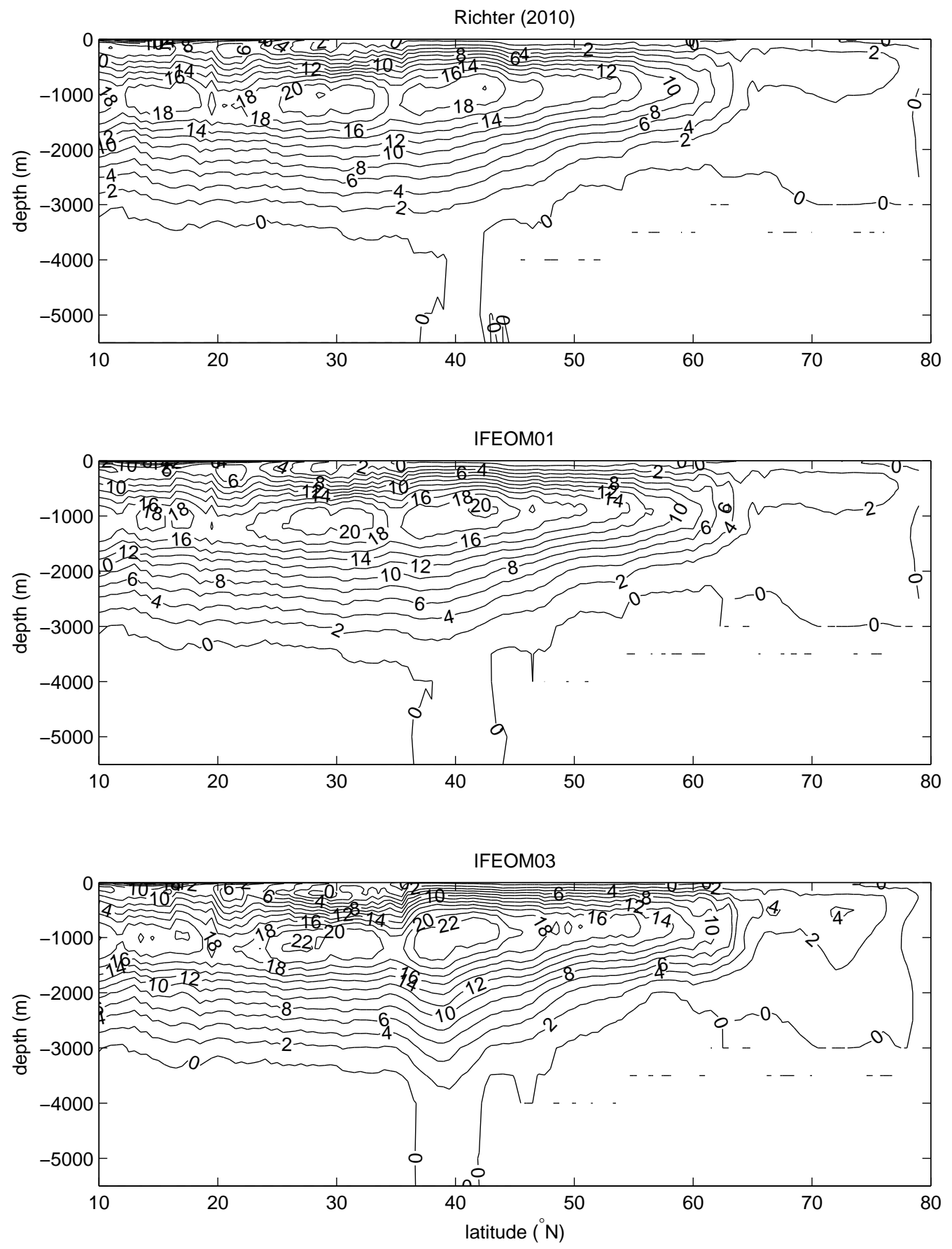

Figure 9: Overturning stream functions (in Sv). 


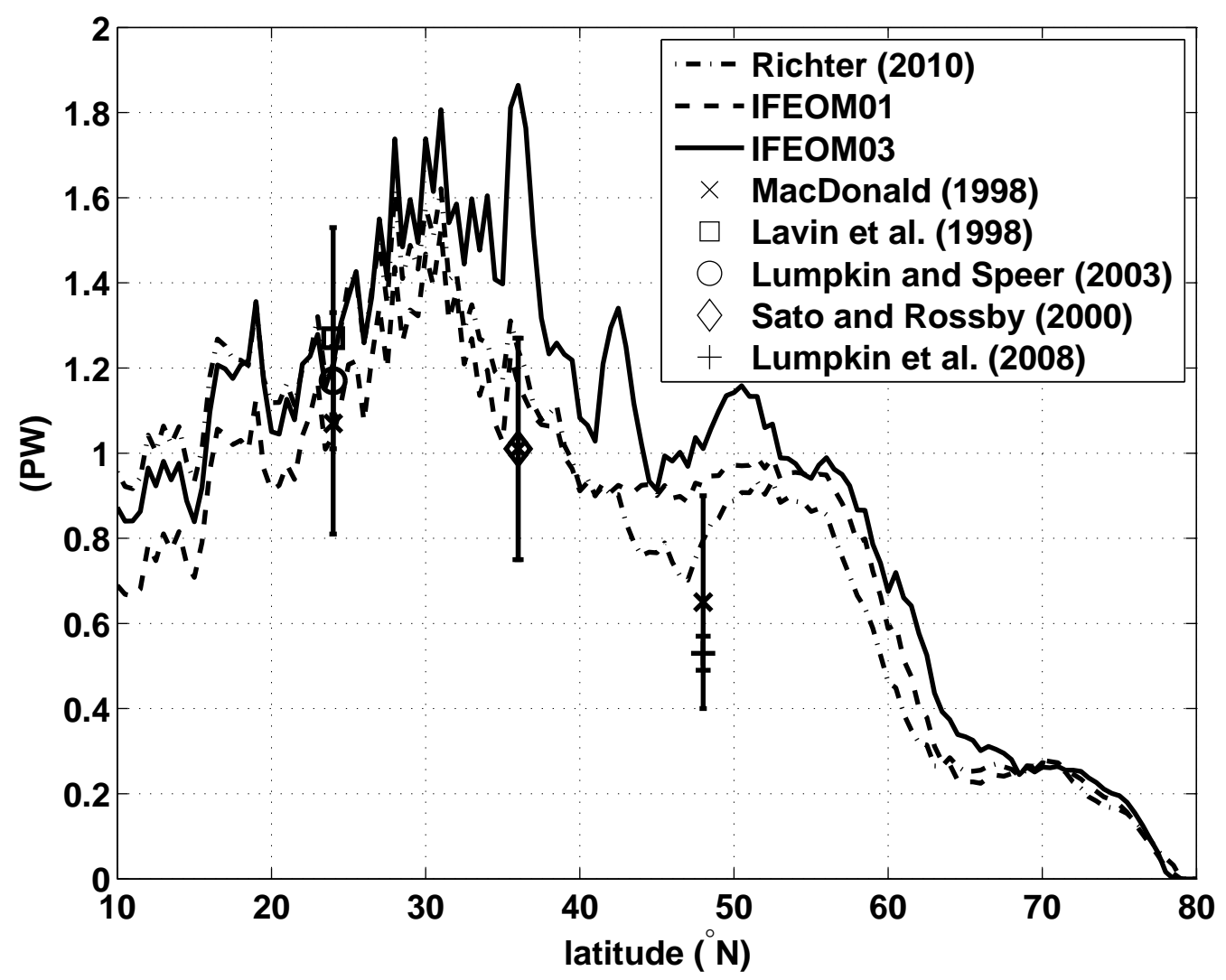

Figure 10: Heat transport estimates across latitudes (in PW), also included are previous estimates from individual section as listed in table 3 .

Estimates of poleward oceanic heat transport differ for the various solutions. While Richter's estimate is tuned to agree within error bars with almost all previous estimates in table 3 (and figure 10), the IFEOM03 solution deviates from the estimates of Macdonald (1998) and Sato and Rossby (2000) for $36^{\circ} \mathrm{N}$ and from estimates of Lumpkin et al. (2008) and Macdonald (1998) for $48^{\circ} \mathrm{N}$. Between latitudes of about $33^{\circ}$ to $68^{\circ} \mathrm{N}$, the IFEOM03 solution transports more heat than established estimates (an incomplete list 


$$
\text { Section Heat transport in }[\mathrm{PW}]
$$

\begin{tabular}{cccc} 
& Richter (2010) & IFEOM01 & IFEOM03 \\
\hline \hline $24^{\circ} \mathrm{N}$ & 1.20 & 1.04 & 1.21 \\
\hline $36^{\circ} \mathrm{N}$ & 1.24 & 1.16 & 1.86 \\
\hline $48^{\circ} \mathrm{N}$ & 0.80 & 0.92 & 1.01
\end{tabular}

Table 2: IFEOM heat transport estimates through zonal sections across the North Atlantic.

is found in table 3 ). There are two distinct peaks near $37^{\circ}$ and $43^{\circ} \mathrm{N}$ that are attributed to the strengthened circulation in the Gulf Stream region. However, IFEOM03 estimates agree within error bars with previous results for low latitudes up to $33^{\circ} \mathrm{N}$, whereas IFEOM01 results are smaller for these lower latitudes (as discussed above).

\section{Conclusions}

Estimates of the mean dynamic topography derived from satellite observations are useful for improving ocean circulation estimates, but only if they have consistent error estimates. The procedure presented in this paper achieves this goal by modeling the MDT on arbitrary ocean model grids as the difference between altimetric sea surface and geoid height in a combined estimation process. As a central feature of this process, the omission error is treated explicitly. Different assumptions about the omission error lead to MDT estimates that are different in resolved signal and estimated error covariance. Assuming little prior knowledge about the omission error 


\begin{tabular}{ccl} 
Section & $\begin{array}{c}\text { Heat transport in } \\
{[\mathrm{PW}] \text { with errors }}\end{array}$ & Source \\
\hline \hline $24^{\circ} \mathrm{N}$ & $1.07 \pm 0.26$ & Macdonald (1998) \\
& $1.27 \pm 0.26$ & Lavin et al. (1998)) \\
& $1.17 \pm 0.08$ & Lumpkin and Speer (2003) \\
\hline $36^{\circ} \mathrm{N}$ & $1.01 \pm 0.26$ & Macdonald (1998) \\
& $1.2 \pm 0.3$ & Sato and Rossby (2000) \\
\hline $48^{\circ} \mathrm{N}$ & $0.65 \pm 0.25$ & Macdonald (1998) \\
& $0.53 \pm 0.04$ & Lumpkin et al. (2008)
\end{tabular}

Table 3: Heat transport estimates of other authors through zonal sections across the North Atlantic.

leads to large uncertainties in the model MDT, while using the EGM08 as the best available estimate of the omission error reduces these uncertainties dramatically.

The design of the estimation process aims at using its products in inverse problems in oceanography. For this purpose the error covariance matrix need not be computed explicitly, but its inverse is used. The inverse error covariance is, by design, given exactly by the normal equations of the estimation problem.

The estimation process works for any target (ocean model) grid to give consistent solutions for ocean modeling. The associated normal equations can be solved exactly, when all observations resolve the grid-scale. For grids finer than the resolution of the satellite observation products, the normal 
equations are singular, but as the inverse of the normal equation matrix is not required for the ocean model inversion, the method can also be applied.

The MDT estimates by the stationary inverse finite element ocean model IFEOM are smooth even when the Rifugio estimate that is used in the inversion is not. This implies that the short scales present in the Rifugio solutions are unphysical, but they are successfully rejected by the ocean model because of the consistent error estimates implicit in the normal equations. This should be interpreted as the main piece of evidence that the assumptions, especially about the omission errors, that went into the geodetic estimation process are consistent with the ocean model. The ocean model IFEOM helps to improve the Rifugio estimates of MDT. The less is assumed about the omission error, the more the MDT estimates benefit from the ocean model contribution.

Fitting IFEOM to the Rifugio MDT generally accelerates the circulation in the model ocean. The model result shows a more pronounced Gulf Stream, increased deep water formation at high latitudes and modified meridional heat transport estimates. Some of these estimates are not consistent with previous estimates. These small discrepancies are attributed to tuning issues in both the geodetic and the oceangraphic estimation procedure and possibly incomplete ocean model dynamics. In this context, stationarity (no time dependence) appears as the main ocean model deficit that needs to be addressed in the future.

Ocean modeling can greatly benefit from space-borne observations. Here, the prospect of consistent satellite-based estimates of MDT with errors on the ocean models grid is put forward in a pilot study. 


\section{Acknowledgements}

This work was funded within the DFG priority programme SPP 1257 "Mass transport and mass distribution in the system Earth". GF and ML thank Falk Richter and Dmitiry Sidorenko for help with IFEOM. Marc Taylor's critical comments improved the manuscript's legibility.

\section{References}

Albertella, A., Rummel, R., 2009. On the spectral consistency of the altimetric ocean and geoid surface: a one-dimensional example. Journal of Geodesy 83, 805-815.

Golub, G.H., van Loan, C.F., 1983. Matrix Computations. John Hopkins University Press, Baltimore, Maryland.

Jekeli, C., 1981. Alternative methods to smooth the Earth's gravity field. Reports of the Department of Geodetic Science. Ohio State University (OSU), Ohio. No. 327.

Jekeli, C., 1996. Spherical harmonic analysis, aliasing, and filtering. Journal of Geodesy 70, 214-223.

Kaula, W.M., 1966. Theory of Satellite Geodesy. Blaisdell Publ. Comp., Massachusetts-Toronto-London.

Kusche, J., 2007. Approximate decorrelation and non-isotropic smoothing of time-variable GRACE-type gravity field models. Journal of Geodesy 81, $733-749$. 
Lavin, A., Bryden, H.L., Parrilla, G., 1998. Meridional transport and heat flux variation in the subtropical North Atlantic. Global Atmosphere-Ocean System 6, 269-293.

Losch, M., Sloyan, B., Schröter, J., Sneeuw, N., 2002. Box inverse models, altimetry and the geoid: Problems with the omission error. Journal of Geophysical Research 107.

Lumpkin, R., Speer, K.G., 2003. Large-scale vertical and horizontal circulation in the North Atlantic Ocean. Journal of Physical Oceanography 33, 1902-1920.

Lumpkin, R., Speer, K.G., Koltermann, K.P., 2008. Transport across $48^{\circ} \mathrm{n}$ in the Atlantic Ocean. Journal of Physical Oceanography 38, 733-752.

Macdonald, A.M., 1998. The global ocean circulation: A hydrographic estimate and regional analysis. Progress in Oceanography 41, 281-382.

Mayer-Gürr, T., Kurtenbach, E., Eicker, A., 2010. ITG-Grace2010 gravity field model. http://www.igg.uni-bonn.de/apmg/index.php?id= itg-grace2010.

MDT_CNES_CLS09, 2009. MDT_CNES_CLS09 was produced by CLS Space Oceanography Division and distributed by Aviso, with support from Cnes (http://www. aviso.oceanobs.com/).

MSS_CNES_CLS10, 2010. MSS_CNES_CLS10 was produced by CLS Space Oceanography Division and distributed by Aviso, with support from Cnes (http://www . aviso.oceanobs.com/). 
Pavlis, N.K., Holmes, S.A., Kenyon, S.C., Factor, J.K., 2008. An Earth Gravitational Model to degree 2160: EGM2008. presented at the 2008 General Assembly of the European Geosciences Union, Vienna, Austria, April 13-18 .

Richter, F., 2010. Nutzung von Argo-Driftern und Satellitenaltimetriedaten zur Ableitung der Zirkulation im Nordatlantik. Ph.D. thesis. Universität Bremen.

Rio, M.H., Hernandez, F., 2004. A mean dynamic topography computed over the world ocean from altimetry, in situ measurements, and a geoid model. Journal of Geophysical Research 109.

Sato, O.T., Rossby, T., 2000. Seasonal and low-frequency variability of the meridional heat flux at $36^{\circ} \mathrm{n}$ in the north atlantic. Journal of Physical Oceanography 30, 606-621.

Schuh, W.D., Becker, S., 2010. Potential field and smoothness conditions, in: Contadakis, M., Kaltsikis, C., Spatalas, S., Tokmakidis, K., Tziavos, I. (Eds.), The apple of knowledge - In honour of Prof. N. Arabelos, University of Thessaloniki. AUTH - Faculty of rural and surveying engineering. pp. $237-250$.

Sidorenko, D., 2004. The North Atlantic circulation derived from inverse models. Ph.D. thesis. Universität Bremen.

Swenson, S., Wahr, J., 2006. Post-processing removal of correlated errors in GRACE data. Geophysical Research Letters 33. 
${ }_{541}$ Wahr, J., Molenaar, M., Bryan, F., 1998. Time-variability of the Earth's 542 gravity field: Hydrological and oceanic effects and their possible detection 543 using GRACE. Journal of Geophysical Research 103, 205-229.

${ }_{544}$ Wunsch, C., Stammer, D., 1998. Satellite altimetry, the marine geoid, and 545 the oceanic general circulation. Ann. Rev. Earth and Planet. Sci. 26, 219$546 \quad 253$. 